\title{
Scottish rehabilitation services: their relationship to socio-economic deprivation
}

\author{
Robin G. McCreadie, Director of Clinical Research and Consultant Psychiatrist; and \\ Douglas J. Williamson, Research Registrar, Crichton Royal Hospital, \\ Dumfries DG1 4TG
}

It is well established that there is a strong relationship between psychiatric admission rates and socioeconomic deprivation; the more deprived the area, the more admissions may be expected (Hirsch, 1988). In the development of community resources for the mentally ill, therefore, an aim might be to site the majority of facilities in areas of greatest social deprivation, as that is where the majority of patients will live. It might also be expected that areas of greater deprivation would have more professional NHS staff. The present survey examines the siting of rehabilitation and support services in the catchment areas of Scottish psychiatric hospitals in relation to measures to socioeconomic deprivation. It also examines the relationship between deprivation and numbers of professional NHS staff in the different catchment areas.

\section{The study}

Psychiatrists provided, first, a complete list of rehabilitation facilities in the catchment areas of 12 Scottish psychiatric hospitals and, second, information about the total numbers of professional NHS staff. Facilities included in-patient rehabilitation units, day hospitals, local authority residential and day units, and voluntary agency residential and day units. The site of each unit was identified by postcode. Jarman indices were used as measures of socioeconomic deprivation (Jarman, 1984). The Jarman score for the relevant postcode sector was taken as the deprivation score for the area in which the rehabilitation facility was sited. A deprivation score was also obtained for each hospital's catchment area by using the scores for the local government districts within it. The latter are themselves 'aggregated' from the scores for individual postcode sectors. The mean number of postcode sectors in the hospitals' catchment areas was 44 (range 10-107) (Information and Statistics Division, Scottish Health Service; Mrs Vera Carstairs, personal communication).

\section{Findings}

The mean Jarman score for the catchment areas of the 12 hospitals $(+14.36$, range +8.14 to +24.17$)$ was similar to that for Scotland as a whole $(+15.59)$. A higher positive score indicates greater deprivation.

Each rehabilitation facility was given a score in the following way. From the Jarman score for the postcode sector in which the facility was sited was subtracted the mean score for the hospital's catchment area. Thus, for example, a day hospital in a postcode sector with a Jarman score of +17.81 in a catchment area with a mean score of +15.92 was given a score of +1.89 . A positive score indicates the facility is in an area of higher deprivation, a negative score that it is in an area of lower deprivation, relative to the mean for that catchment area.

The scattergram (Fig. 1) shows the distribution of hospital based rehabilitation units $(n=12)$, hospital

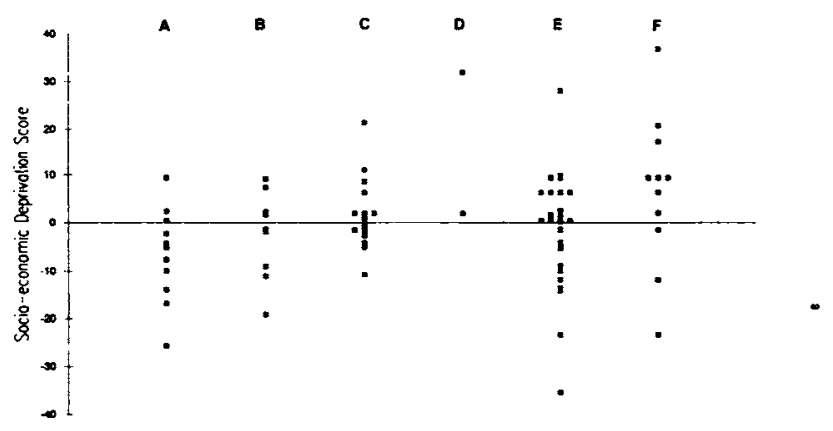

FIG. 1. Socio-economic deprivation scores for rehabilitation facilities in 12 catchment areas.

$A=$ Hospital based rehabilitation units; $B=$ NHS hospital-based and community day units; $C=$ Local authority residential units; $\mathrm{D}=$ Local authority day units; $\mathrm{E}=$ Voluntary agency residential units; $\mathrm{F}=$ Voluntary agency day units. See text for derivation of scores. 
based and community day hospitals $(n=10)$, local authority residential $(n=18)$ and day $(n=2)$ units, and voluntary organisation residential $(n=30)$ and day $(n=10)$ units. It can be seen that 42 of the 82 $(51 \%)$ units were in areas more deprived than the catchment average, $7(9 \%)$ were in a postcode area more than +10 Jarman points from the average, and $13(16 \%)$ were more than -10 Jarman points from the average. These latter units included three hospital based rehabilitation units, two NHS day units, one local authority residential unit, five voluntary organisation residential units and two voluntary organisation day centres.

Correlations were measured between Jarman scores for catchment areas and numbers of NHS professional staff in these areas (expressed as numbers per 100,000 of the general population). The correlation co-efficient, $r$, between deprivation and numbers of consultants was +0.32 , trained nurses +0.03 , community psychiatric nurses 0.00 , trained occupational therapists $+0.58,(P<0.05)$, and clinical psychologists -0.10 . In 11 of the 12 catchment areas it was also possible to measure the correlation between the Jarman score for the area and, first, the total number of residential places for the long term mentally ill in the area $(r=+0.12)$; and, second, the total number of day places $(r=+0.47$, not statistically significant).

\section{Comment}

The results must be interpreted with caution for at least two reasons. First, information was obtained about the rehabilitation facilities in the catchment areas of only 12 hospitals. These hospitals may not be typical of Scottish psychiatric hospitals as a whole. However, the mean deprivation score of the catchment areas was very similar to the Scottish mean. Secondly, Jarman deprivation scores are derived from the 1981 United Kingdom census. No doubt there will have been a shift in socio-economic deprivation in some postcode sectors; how great this shift has been, however, cannot be determined until the detailed results of the 1991 census are known.

The results did not show that rehabilitation facilities have been developed especially in the more deprived parts of a hospital's catchment area. In the 12 hospitals studied there was an approximately normal distribution of facilities throughout the catchment areas, with a possible 'tail' extending into areas of low deprivation. This was due first to the hospitals themselves, many of which were originally built on green field sites. With the passage of time private housing has developed and encircled them. Second, a number of hostels and day centres run by voluntary organisations were in areas of low social deprivation. Probably these facilities were in large houses, formerly privately owned in residential areas.

Do patients from more deprived parts of the catchment area using facilities in less deprived areas feel 'out of place'? If day attenders, do they resent the travelling that may be involved? Would they rather hostels and day centres were in more disadvantaged parts of the catchment area? Or do they welcome the opportunity to get away from a poorer environment? The views of 'consumers' would be of considerable interest.

On a national scale, that is when betweencatchment area differences were examined, there was little evidence that professional staffing in general, and accommodation or day care for the long-term mentally ill were more often to be found in the more deprived catchment areas. It is probable that psychiatric services within each Scottish health board are fighting for their share of the 'cake' allocated to that board; and their success (or failure) is little influenced by the socio-economic characteristics of the area.

We conclude by suggesting that when the NHS and voluntary organisation plan future residential and day units, they should take into account the socio-economic characteristics of the area in which they propose to site the facilities.

\section{Acknowledgements}

This study was carried out under the auspices of the Rehabilitation Group, Scottish Division, Royal College of Psychiatrists. We gratefully acknowledge the help of the many professionals in the psychiatric hospitals and units who helped to supply information and to Dr J. G. Greene for statistical advice.

\section{References}

HiRsch, S. R. (1988) Psychiatric Beds and Resources: Factors Influencing Bed Use and Service Planning. Report of a Working Party of the Section of Social and Community Psychiatry of the Royal College of Psychiatrists. London: Gaskell.

JARMAN, B. (1984) Identification of underprivileged areas: validation and distribution of scores. British Medical Journal, 289, 1587-1592. 